\title{
Parvovirus B19 infection: fifth disease and more
}

Epidemiology and background: Parvovirus B19 is a member of the large Parvoviridae family, which includes pathogenic animal viruses that have long been of interest to veterinarians and virologists. ${ }^{1}$ The discovery of a parvovirus in human blood in the mid 1970s led to study of the epidemiology and pathogenesis of this common childhood infection. The reliance of this virus on human erythroid progenitor cells for propagation and its highly immunogenic capsid explain the clinical spectrum and populations susceptible to the complications of parvovirus B19 infection.

The only known natural host cell of parvovirus B19 is the human erythroid progenitor. Erythropoiesis is probably temporarily suppressed in all B19 infections, since in experimental settings reticulocyte counts in previously healthy seronegative volunteers have been observed to temporarily drop to zero a week or so after inoculation. ${ }^{2}$ Hemoglobin levels ordinarily remain stable because the erythrocyte has a long half-life. In most people the virus is highly immunogenic, and at the time of presentation almost all patients have detectable IgM antibodies, which persist for 2 to 3 months after acute infection. Antibody production is correlated with the disappearance of the virus from the bloodstream, and IgG production appears to confer lasting immunity. Infection is global and very common. About half of 15-year-olds have specific antiparvovirus B19 antibodies. Uninfected adults remain susceptible, and most become infected and seropositive during the course of their lifetimes. ${ }^{1}$

In temperate climates the infection usually occurs in spring. The virus is spread by respiratory droplets, and transmission most often occurs during the week before the onset of symptoms. The incubation period is
4-21 days. In children the infection typically causes erythema infectiosum, or "fifth disease," so-called because it was the fifth of the common rash-associated diseases of childhood to be identified. It presents with fever, malaise, red ("slapped") cheeks, followed 4-14 days later with a lacy, itchy full-body rash that can last up to 3 weeks. In adults the rash is often absent, but arthropathy, which tends to be symmetrical and involve multiple joints, is more common, particularly among middle-aged women. Both the rash and the arthropathy are thought to result from the deposition of immune complexes. The illness is self-limiting, and most healthy people recover with lasting immunity to reinfection. ${ }^{1}$

Certain populations are vulnerable to complications from the interrupted erythropoiesis that arises with infection. These populations include people with hematologic disorders that cause an increased rate of erythrocyte destruction, such as hereditary spherocytosis and sickle cell disease. The fetus is also at risk. The risk of transplacental infection among women who are infected during pregnancy is about $30 \%$. Parvovirus infects the fetal liver, the main site of erythrocyte production during early development, and can induce severe anemia and perhaps myocarditis, both of which may lead to congestive heart failure ("hydrops fetalis"). There is a $5 \%-9 \%$ risk of fetal loss among infected pregnant women; the risk appears to be greatest in the second trimester. ${ }^{1}$

People who have a high demand for erythrocyte production are at risk of experiencing severe anemia or a transient aplastic crisis. In addition, people who are unable to generate an adequate antibody response, such as people with HIV infection, are at risk of persistent parvovirus infection.
Clinical management: Most healthy infected people do not need to consult a physician. Laboratory diagnosis can be made on the basis of serologic testing (IgM-specific antibodies). DNA assays may be required in immunocompromised patients who are unable to mount an antibody response. Although there is no specific therapy, immunocompromised patients may benefit from immune globulin, and anemic patients may require transfusion or even hematopoietic stem-cell transfer. Pregnant women exposed to infection should have their immunity tested.

Prevention: There is no need to keep infected children away from school, since in most people this illness is mild and selflimiting, and most children are no longer infective by the time the rash appears and the diagnosis is made. Frequent hand washing can reduce the spread of respiratory droplets. The US Centers for Disease Control and Prevention does not recommend that pregnant women be routinely excluded from a workplace where an outbreak is occurring. ${ }^{3}$ The decision to stay away is a personal decision made after the woman's discussions with her family, doctor and employer.

The development of an effective vaccine is technically feasible but has been stalled primarily by a lack of commercial interest.

\section{Erica Weir \\ CMAF}

\section{References \\ 1. Young NS, Brown KE. Parvovirus B19. N Engl 7 Med 2004;350:586-97. \\ 2. Anderson MJ, Higgins PG, Davis LR, Willman JS, Jones SE, Kidd IM, et al. Experimental parvoviral infection in humans. F Infect Dis 1985;152:257-65. \\ 3. Parvovirus B19 infection and pregnancy. Atlanta: US Centers of Disease Control and Prevention. Available: www.cdc. gov/ncidod/dvrd/revb/respiratory/B19 \&preg.htm (accessed 8 Feb 05).}

